\title{
On Maximum Lee Distance Codes
}

\author{
Tim L. Alderson and Svenja Huntemann \\ Department of Mathematical Sciences, University of New Brunswick Saint John, Saint John, NB, Canada E2L 4L5
}

Correspondence should be addressed to Tim L. Alderson; tim@unb.ca

Received 25 September 2012; Accepted 21 November 2012

Academic Editor: Annalisa de Bonis

Copyright (C) 2013 T. L. Alderson and S. Huntemann. This is an open access article distributed under the Creative Commons Attribution License, which permits unrestricted use, distribution, and reproduction in any medium, provided the original work is properly cited.

\begin{abstract}
Singleton-type upper bounds on the minimum Lee distance of general (not necessarily linear) Lee codes over $\mathbb{Z}_{q}$ are discussed. Two bounds known for linear codes are shown to also hold in the general case, and several new bounds are established. Codes meeting these bounds are investigated and in some cases characterised.
\end{abstract}

\section{Introduction}

The Lee metric was introduced by Lee [1] in 1958 as an alternative to the Hamming metric for certain noisy channels. It found application and in particular was later developed for certain noisy channels (primarily those using phase-shift keying modulation [2]). The past decade has witnessed a burst of new and varied applications for codes defined in the Lee metric (Lee codes) including constrained and partialresponse channels [3], interleaving schemes [4], orthogonal frequency-division multiplexing [5], multidimensional burst-error correction [6], and error correction for flash memories [7]. These recent applications give increased interest in questions surrounding optimal Lee codes.

Similar to the case of the Hamming metric, it is desirable to investigate upper bounds on the minimum Lee distance of a code given the code size, code length, and alphabet size. Codes meeting these bounds are of special interest as they are optimal in the sense that their minimum distance is largest. Under the Hamming metric, such codes are referred to as maximum distance separable (MDS) codes. Under the Lee metric, such codes may be referred to as Maximum Lee Distance Separable (MLDS) codes. Here, we will present several upper bounds similar to the Singleton bound and investigate the existence question of MLDS codes. In certain cases, we are able to completely characterize MLDS codes.

\section{Preliminaries}

An $(n, k, d)_{q}$ block code $C$ is a collection of $q^{k} n$-tuples (codewords) over an alphabet of size $q$ such that the minimum
(Hamming) distance between any two codewords is $d$ (hence, no two codewords have as many as $n-d+1$ common coordinates). Here, $k=\log _{q}|C|$ is the dimension of $C$, which need not be an integer. Where context demands, we may also denote the Hamming distance by $d_{H}$. The Singleton bound states that

$$
d \leq n-\lceil k\rceil+1
$$

and holds for all block codes. Codes meeting this bound with equality are called maximum distance separable (MDS) codes. Research on both linear and nonlinear MDS codes has been extensive (e.g., see [8-10] and references therein).

2.1. Lee Codes. Let $\mathbb{Z}_{q}=\{0,1, \ldots, q-1\}$ be the set of representatives of the integer equivalence classes modulo $q$. The Lee weight of any element $a \in \mathbb{Z}_{q}$ is given by $w_{L}(a)=\min \{a, q-$ a\}. Given an element $c=\left(c_{1}, c_{2}, \ldots, c_{n}\right) \in \mathbb{Z}_{q}^{n}$, the Lee weight of $c$, denoted $w_{L}(c)$, is given by

$$
w_{L}(c)=\sum_{i=1}^{n} \min \{a, q-a\} .
$$

For $c, e \in \mathbb{Z}_{q}^{n}$, the Lee distance $d_{L}(c, e)$ between $c$ and $e$ is defined to be the Lee weight of their difference,

$$
\begin{aligned}
d_{L}(c, e) & =w_{L}(c-e) \\
& =\sum_{i=1}^{n} \min \left\{c_{i}-e_{i}(\bmod q), e_{i}-c_{i}(\bmod q)\right\} .
\end{aligned}
$$


A Lee code $C$ will be specified by $\left(n, k, d_{L}\right)_{q}$, where $n, k$, and $q$ are as aforementioned, and $d_{L}$ is the minimum Lee distance of $C$; that is, $d_{L}=\min \left\{d_{L}(c, e) \mid c, e \in C, c \neq e\right\}$.

Observe that in the case that $q=2$ or 3 (binary or ternary codes), the Hamming and Lee metrics are identical. Thus a code $C$ with $q=2$ or $q=3$ is MLDS if and only if $C$ is MDS. As much literature discusses MDS codes, we shall focus attention on the cases $q>3$.

A code $C$ over the alphabet $\mathbb{Z}_{q}$ is considered linear if $C$ forms a submodule of $\mathbb{Z}_{q}^{n}$. Unless otherwise stated, we do not assume linearity.

2.2. Code Equivalence. We shall say a permutation $\sigma$ of $\mathbb{Z}_{q}$ is a Lee permutation if it preserves Lee distance. That is, $d_{L}(a, b)=$ $d_{L}(\sigma(a), \sigma(b))$, for all $a, b \in \mathbb{Z}_{q}$. For example, any translation $\sigma(x)=x+t(\bmod q)$ is a Lee permutation.

Given an $\left(n, k, d_{L}\right)_{q}$ Lee code $C$, we may define the following operations on codewords. A positional permutation is a permutation (on $n$ letters) of the coordinate positions, applied to each word of C. A Lee symbol permutation is a Lee permutation applied to a fixed coordinate position throughout $C$. Two codes are equivalent if one may be obtained from the other by applying a sequence of Lee symbol or positional permutations.

Example 1. The code

$$
C=(0,0) \quad(1,2) \quad(2,4) \quad(3,1) \quad(4,3)
$$

is a $(2,1,3)_{5}$ Lee code. The equivalent codes may be produced by applying the Lee permutation $(0)(14)(23)$ to one or more of the coordinate entries of $C$. For example, the code

$$
C^{\prime}=(0,0) \quad(1,3) \quad(2,1) \quad(3,4) \quad(4,2)
$$

is equivalent to $C$.

It follows that any given Lee Code is equivalent to a Lee Code containing the zero codeword. This observation shall prove quite useful in the sequel.

\section{Singleton-Type Bounds}

In 2000 Shiromoto, [11] proved the following upper bound for linear codes over $\mathbb{Z}_{q}$.

Proposition 2 (see [11]). If $C$ is a linear $\left(n, k, d_{L}\right)_{q}$ Lee code over $\mathbb{Z}_{q}$, then

$$
\left\lfloor\frac{d_{L}-1}{\lfloor q / 2\rfloor}\right\rfloor \leq n-k
$$

We shall show that bound (6) holds for general (not necessarily linear) Lee codes. Indeed, observe that for a given code, any two codewords differ in at most $d_{H}$ coordinate positions, from which it follows that $d_{L} \leq\lfloor q / 2\rfloor d_{H}$. Combining this observation with the Singleton bound (1) gives the following.
Proposition 3. For any $\left(n, k, d_{L}\right)_{q}$ Lee code C, the following holds:

$$
d_{L} \leq\left\lfloor\frac{q}{2}\right\rfloor(n-\lceil k\rceil+1) .
$$

Remark 4. Observe that

$$
\begin{aligned}
d_{L} & \leq\left\lfloor\frac{q}{2}\right\rfloor(n-\lceil k\rceil+1) \Longrightarrow\left\lfloor\frac{d_{L}-1}{\lfloor q / 2\rfloor}\right\rfloor \\
& \leq\left\lfloor n-\lceil k\rceil+\frac{\lfloor q / 2\rfloor-1}{\lfloor q / 2\rfloor}\right\rfloor \\
& =n-\lceil k\rceil .
\end{aligned}
$$

Consequently, Proposition 3 subsumes the bound of Shiromoto (6).

Remark 5. Suppose that $C$ is an $\left(n, k, d_{L}\right)_{q}$ Lee code with $\lceil k\rceil=1$ meeting bound (7), so that $d_{L}=\lfloor q / 2\rfloor n$. Assume that $C$ contains the zero codeword. It follows that $C$ contains precisely two codewords. Moreover, the nonzero codeword has (Lee) weight $d_{L}$. Hence, if $q$ is even, then the nonzero codeword has all entries $q / 2$. If $q$ is odd, then the nonzero codeword has each entry being either $(q+1) / 2$ or $(q-1) / 2$.

We shall make improvements to bound (7) under various settings. Observe that codes for which $n<k$ necessarily contain repeated codewords; whence, $d_{L}=0$. Also, codes with $n=k$ necessarily comprise the entire space $\mathbb{Z}_{q}^{n}$, and consequently $d_{L}=1$. Hence, in the sequel, we shall focus on codes with $n>k$.

3.1. Bounds on Codes over Even-Sized Alphabets. By restricting to codes over even-sized alphabets, we obtain the following bound.

Theorem 6. For any Lee metric code with alphabet size q even, of length $n$ and minimum Lee distance $d_{L}$, the following holds:

$$
d_{L} \leq \frac{q}{2} n-\left\lceil\log _{2}|C|\right\rceil+1
$$

Proof. If $q$ is even, we can create a distance preserving map (Gray code) $\phi$ (similar to that used in $[12,13]$ ) from the metric space $\left(\mathbb{Z}_{q}, d_{L}\right)$ to the metric space $\left(\mathbb{Z}_{2}^{q / 2}, d_{H}\right)$ defined by

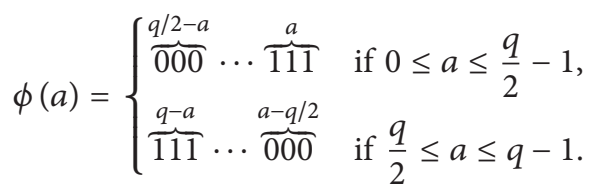

For $c \in C$, we extend the map coordinatewise; thus, $\phi(c)=$ $\left(\phi\left(c_{1}\right) \cdots \phi\left(c_{n}\right)\right)$, and let $C^{\prime}=\phi(C)=\{\phi(c) \mid c \in C\}$. It follows that $C^{\prime}$ is a binary code of length $n(q / 2)$ with $|C|=\left|C^{\prime}\right|$, and $d_{L}(C)=d_{H}\left(C^{\prime}\right)$. The result follows from the Singleton bound (1). 
3.1.1. Special Cases: Characterizing and Improving. If restrictions are made on the size of $C$, we are able to characterize cases of equality in bound (9). The following well known property of binary MDS codes shall be of use. See, for example, [14].

Lemma 7. If $C$ is a binary $(n, k, d)_{2}$ MDS code of integral dimension $k \geq 1$, then either

(1) $k=1$, and $C$ is the repetition code $\{(0,0, \ldots, 0)$, $(1,1, \ldots, 1)\}$,

(2) $k \geq 2, n=k+1$, and $C$ is the binary parity check code consisting of all binary $n$-tuples of even (Hamming) weight, or

(3) $k \geq 2, n=k$, and $C$ is the entire space $\mathbb{Z}_{2}^{n}$.

Lemma 8. Let $C$ be an $\left(n, k, d_{L}\right)_{q}$ Lee code with $n>k \geq 1$, $q>2$ even, and $\log _{2}|C| \in \mathbb{Z}$. If $d_{L}=(q / 2) n-\log _{2}|C|+1$, then either

(1) $k=1$, and $C$ is the repetition code $\{(00 \cdots 0) \quad((q / 2)(q / 2) \cdots q / 2)\}$, or

(2) $k=n-(1 / 2)$, and $q=4$.

Proof. Assume that $d_{L}=(q / 2) n-\log _{2}|C|+1$. Let $\phi$ be as defined in the proof of Theorem 6 . Then, $C^{\prime}=\phi(C)$ is a binary $\left(n^{\prime}, k^{\prime}, d^{\prime}\right)$ MDS code, $n^{\prime}=(q / 2) n$, and $k^{\prime}=\log _{2}|C|$. Note that since $n \geq 2$ and $q \geq 4$, we have $n^{\prime} \geq 4$. According to Lemma 7 , we have three cases to consider.

Case $1\left(k^{\prime}=1\right)$. In this case, $C^{\prime}$ is the binary repetition code; hence, $C$ is the code

$$
(00 \cdots 0) \quad\left(\frac{q}{2} \frac{q}{2} \cdots \frac{q}{2}\right)
$$

Case $2\left(k^{\prime}>1, n^{\prime}=k^{\prime}+1\right)$. In this case, $C^{\prime}$ is the binary parity check code of length $n^{\prime}$. As such, the vector $(101000 \cdots 0)$ is in $C^{\prime}$. From the definition of $\phi$, it follows that $q / 2 \leq 2$; so, $q=4$.

Note that since $q=4$, we have $n^{\prime}=2 n=k^{\prime}+1=2 k+1$.

Case $3\left(k^{\prime}>1, n^{\prime}=k^{\prime}\right)$. In this case, $C^{\prime}=\mathbb{Z}_{2}^{k^{\prime}}$. Again, the vector $(101000 \cdots 0)$ is in $C^{\prime}$, giving $q=4$. But then, $n^{\prime}=2 n=$ $k^{\prime}=2 k$, contradicting $n>k$.

Remark 9. An example of a code satisfying item (2) in Lemma 8 is the $(2,3 / 2,2)_{4}$ Lee code:

$$
C=\{(00),(02),(20),(22),(13),(31),(11),(33)\} \text {. }
$$

With Lemma 8, we see that the bound in Theorem 6 may be improved in certain cases.

Theorem 10. Let $C$ be an $\left(n, k, d_{L}\right)_{q}$ Lee code with $q>4$ even, $n>k \geq 1$, and $\log _{2}|C| \in \mathbb{Z}$. Then,

$$
d_{L} \leq \frac{q}{2} n-\log _{2}|C|
$$

3.2. Bounds on General Alphabets. In this section, we shall make improvements to the bound in Proposition 3. First, some intermediary lemmata are introduced.

Lemma 11. If $C$ is an $\left(n, k, d_{L}\right)_{q}$ Lee code with $d_{L} \geq\lfloor q / 2\rfloor(n-$ $\lfloor k\rfloor)+1$, then the following hold:

(1) $k$ is an integer;

(2) Fix $k$ coordinate positions $i_{1}<i_{2}<\cdots<i_{k}$, and $k$ (not necessarily distinct) elements $\alpha_{1}, \ldots, \alpha_{k} \in \mathbb{Z}_{q}$. Then, there exists precisely one codeword $c$ having $c_{i_{j}}=\alpha_{j}$, $j=1,2, \ldots, k$.

Proof. For the first part, observe that if two codewords agree in $\lfloor k\rfloor$ coordinates, then their distance is at most $\lfloor q / 2\rfloor(n-\lfloor k\rfloor)$, violating the minimum Lee distance condition. Consequently, $|C| \leq q^{\lfloor k\rfloor}$, giving $k=\lfloor k\rfloor$.

For the second part, fix $k$ coordinate positions $\left\{i_{1}, \ldots, i_{k}\right\}$. The result then follows by observing that no two codewords agree in all of these positions, and there are $q^{k}$ codewords in total.

Lemma 12. If $C$ is $a(k+1, k, 3)_{5}$ Lee code, $k \in \mathbb{Z}$, then $k=1$.

Proof. First, note that from Example 1, a $(2,1,3)_{5}$ Lee code does in fact exist.

Now, let $k \geq 2$, and assume without loss of generality that the zero codeword is in $C$. Consider (Lemma 11) the three codewords $w_{1}, w_{2}$, and $w_{3}$, having first $k$ coordinates $100 \cdots 0$, $0100 \cdots 0$, and $400 \cdots 0$, respectively. In order to maintain minimum Lee distance 3 from the zero codeword, the $(k+1)$ st coordinate of each $w_{i}$ must be from $\{2,3\}$. Thus, at least two of $\left\{w_{1}, w_{2}, w_{3}\right\}$ agree in the final coordinate, violating the minimum distance property.

Lemma 13. If $C$ is an $\left(n, k, d_{L}\right)_{q}$ code with $q>3$, and integral dimension $k>1$, then $d_{L} \leq\lfloor q / 2\rfloor(n-k)$.

Proof. Suppose that $C$ is an $\left(n, k, d_{L}\right)_{q}$ code, $q>3,1<k \in$ $\mathbb{Z}$, with $d_{L} \geq\lfloor q / 2\rfloor(n-k)+1$. From Lemma 11, there exist codewords $a, b$, and $c$ having first $k-1$ coordinates 0 and $k$ th coordinate 0,1 , and $q-1$, respectively. Consequently, we have $d_{L}=\lfloor q / 2\rfloor(n-k)+1$.

If $q$ is even, then $d_{L}(a, b)=(q / 2)(n-k)+1$ gives $b_{i}=$ $a_{i}+(q / 2)(\bmod q)$, for $i=k+1, k+2, \ldots, n$. Similarly, $c_{i}=a_{i}+$ $(q / 2)(\bmod q)$, for $i=k+1, k+2, \ldots, n$. But then, $d_{L}(b, c)=2$, contradicting the condition $d_{L}=(q / 2)(n-k)+1$.

If $q$ is odd, then a similar argument shows that for each of the final $(n-k)$ coordinate, $d_{L}\left(b_{i}, c_{i}\right) \leq 1$. Consequently, $d_{L}(b, c) \leq n-k+2$. Therefore, we have $n-k+2 \geq\lfloor q / 2\rfloor(n-k)+1$ from which it follows that $q=5, n=k+1$, and $d=3$. From Lemma 12, it then follows that $k=1$, a contradiction.

From Lemmas 11 and 13, we have the following result.

Theorem 14. If $C$ is an $\left(n, k, d_{L}\right)_{q}$ Lee code $q>3, n>k>1$ then

$$
d_{L} \leq\left\lfloor\frac{q}{2}\right\rfloor(n-\lfloor k\rfloor) .
$$


3.3. Bound Based on Plotkin's Average Distance Bound. Based on the fact that the minimum distance between pairs of codewords cannot exceed the average distance between all pairs of distinct codewords, Wyner and Graham [15] obtained the following bound.

Proposition 15. If $C$ is an $\left(n, k, d_{L}\right)_{q}$ Lee code, then

$$
d_{L} \leq \frac{n \bar{D}}{1-(1 /|C|)},
$$

where $\bar{D}$ is the average Lee weight of $\mathbb{Z}_{q}$, given by

$$
\bar{D}= \begin{cases}\frac{q^{2}-1}{4 q} & \text { if } q \text { is odd } \\ \frac{q}{4} & \text { if } q \text { is even. }\end{cases}
$$

Using the previous result, we may establish a further Singleton-type bound for Lee codes.

Theorem 16. Let $C$ be an $\left(n, k, d_{L}\right)_{q}$ code. Let $\alpha$ be the maximal number of codewords mutually agreeing in $\lceil k\rceil-1$ common coordinates. Then,

$$
d_{L} \leq\lfloor(n-\lceil k\rceil+1) \cdot a\rfloor
$$

where

$$
a= \begin{cases}\frac{q^{2}-1}{4 q} \cdot \frac{\alpha}{\alpha-1} & \text { if qodd, } \\ \frac{q}{4} \cdot \frac{\alpha}{\alpha-1} & \text { if } q \text { even. }\end{cases}
$$

Proof. Let $C$ be an $\left(n, k, d_{L}\right)_{q}$ code, and let $\alpha$ be as defined earlier. Consider a collection $C_{1}$ of $\alpha$ codewords mutually agreeing in $\lceil k\rceil-1$ coordinates, assumed to be the first $\lceil k\rceil-1$ coordinates. Let $C^{\prime}$ be the corresponding punctured code obtained by deleting the first $\lceil k\rceil-1$ coordinates of each word in $C_{1}$.

It follows that $C^{\prime}$ is an $\left(n-\lceil k\rceil+1,1, d^{\prime}\right)_{q}$ code with $d_{L}^{\prime} \geq$ $d_{L}$. Applying Proposition 15 to $C^{\prime}$, we obtain

$$
d_{L} \leq d_{L}^{\prime} \leq \frac{(n-\lceil k\rceil+1) \bar{D}}{1-(1 / \alpha)} .
$$

Finally, applying (16), we get the result.

Suppose that $C$ is an $\left(n, k, d_{L}\right)_{q}$ Lee code, and $\alpha$ is as defined ealier. Observe that since $|C|>q^{\lceil k\rceil-1}$, it follows that $\alpha \geq 2$. So, if $q$ is even, the bound in Theorem 16 always meets or exceeds bound (7). Moreover, if $k \in \mathbb{Z}$, simple counting shows that $\alpha \geq q$. This gives the following corollary, much improving bound (7).

Corollary 17. If $C$ is an $\left(n, k, d_{L}\right)_{q}$ Lee code with $k \in \mathbb{Z}$, then

$$
d_{L} \leq(n-k+1) \cdot a,
$$

where

$$
a= \begin{cases}\frac{q^{2}}{4(q-1)} & \text { if } q \text { even }, \\ \frac{q+1}{4} & \text { if } q \text { odd } .\end{cases}
$$

Remark 18. Chiang and Wolf [16] established the bound in Corollary 17 for linear codes.

Codes meeting the bounds in Theorem 16 do exist [16]. Two examples follow:

(1) for $p$ as prime, the linear one-dimensional (equidistant) $(p-1,1,((p-1) / 2) \cdot 3)_{p}$ code generated by $(1,2, \ldots, p-1)$;

(2) the linear $(4,2,4)_{5}$ Lee code with generator matrix

$$
G=\left[\begin{array}{llll}
3 & 4 & 1 & 0 \\
0 & 3 & 4 & 1
\end{array}\right]
$$

\section{Conclusion}

Several upper bounds on the minimum Lee distance of a block code similar to the Singleton bound were established. Codes actually meeting some of these bounds were presented. Two bounds known for linear codes were shown (see Proposition 2 and Corollary 17) to hold for nonlinear codes. Several new bounds were also established.

\section{Acknowledgments}

The first author thanks the NSERC of Canada for support through the Discovery Grants Program. The second author thanks the NSERC of Canada for support through the Undergraduate Student Research Awards Program.

\section{References}

[1] C. Y. Lee, "Some properties of nonbinary error-correcting codes," IEEE Transactions on Information Theory, vol. 4, no. 2, Article ID 012263, pp. 77-82, 1958.

[2] K. Nakamura, "A class of error correcting codes for DPSK channels," in Proceedings of the International Conference on Communications (ICC '79), vol. 3, p. 45, 1979.

[3] R. M. Roth and P. H. Siegel, "Lee-metric BCH codes and their application to constrained and partial-response channels," IEEE Transactions on Information Theory, vol. 40, no. 4, pp. 10831096, 1994.

[4] M. Blaum, J. Bruck, and A. Vardy, "Interleaving schemes for multidimensional cluster errors," IEEE Transactions on Information Theory, vol. 44, no. 2, pp. 730-743, 1998.

[5] K.-U. Schmidt, "Complementary sets, generalized Reed-Muller codes, and power control for OFDM," IEEE Transactions on Information Theory, vol. 53, no. 2, pp. 808-814, 2007.

[6] T. Etzion and E. Yaakobi, "Error-correction of multidimensional bursts," IEEE Transactions on Information Theory, vol. 55, no. 3, pp. 961-976, 2009.

[7] A. Barg and A. Mazumdar, "Codes in permutations and error correction for rank modulation," IEEE Transactions on Information Theory, vol. 56, no. 7, pp. 3158-3165, 2010.

[8] J. W. P. Hirschfeld, G. Korchmaros, and F. Torres, Algebraic Curves over a Finite Field, Princeton Series in Applied Mathematics, Princeton University Press, Princeton, NJ, USA, 2008.

[9] T. L. Alderson, A. A. Bruen, and R. Silverman, "Maximum distance separable codes and arcs in projective spaces," Journal of Combinatorial Theory A, vol. 114, no. 6, pp. 1101-1117, 2007. 
[10] S. Ball, "On sets of vectors of a finite vector space in which every subset of basis size is a basis," Journal of the European Mathematical Society, vol. 14, no. 3, pp. 733-748, 2012.

[11] K. Shiromoto, "Singleton bounds for codes over finite rings," Journal of Algebraic Combinatorics, vol. 12, no. 1, pp. 95-99, 2000.

[12] S. T. Dougherty and C. Fernandez-Cordoba, "Codes over $\mathbb{Z}_{2^{k}}$, Gray map and self-dual codes," Advances in Mathematics of Communications, vol. 5, no. 4, pp. 571-588, 2011.

[13] S. T. Dougherty and K. Shiromoto, "Maximum distance codes over rings of order 4," IEEE Transactions on Information Theory, vol. 47, no. 1, pp. 400-404, 2001.

[14] F. J. MacWilliams and N. J. A. Sloane, The Theory of ErrorCorrecting Codes. I, vol. 16 of North-Holland Mathematical Library, North-Holland Publishing, Amsterdam, The Netherland, 1977.

[15] A. D. Wyner and R. L. Graham, "An upper bound on minimum distance for a k-ary code," Information and Control, vol. 13, no. 1, pp. 46-52, 1968.

[16] J. C. Chiang and J. K. Wolf, "On channels and codes for the Lee metric," Information and Control, vol. 19, no. 2, pp. 159-173, 1971. 


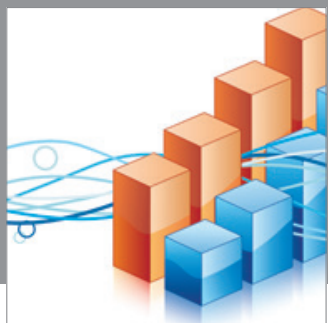

Advances in

Operations Research

mansans

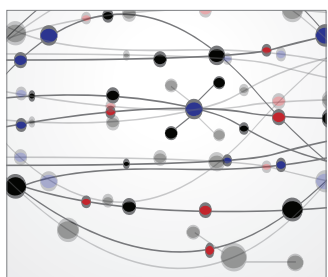

The Scientific World Journal
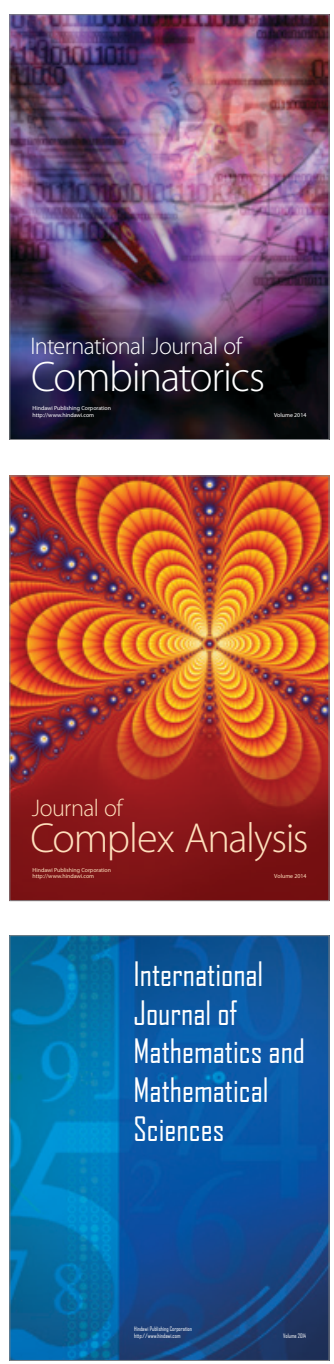
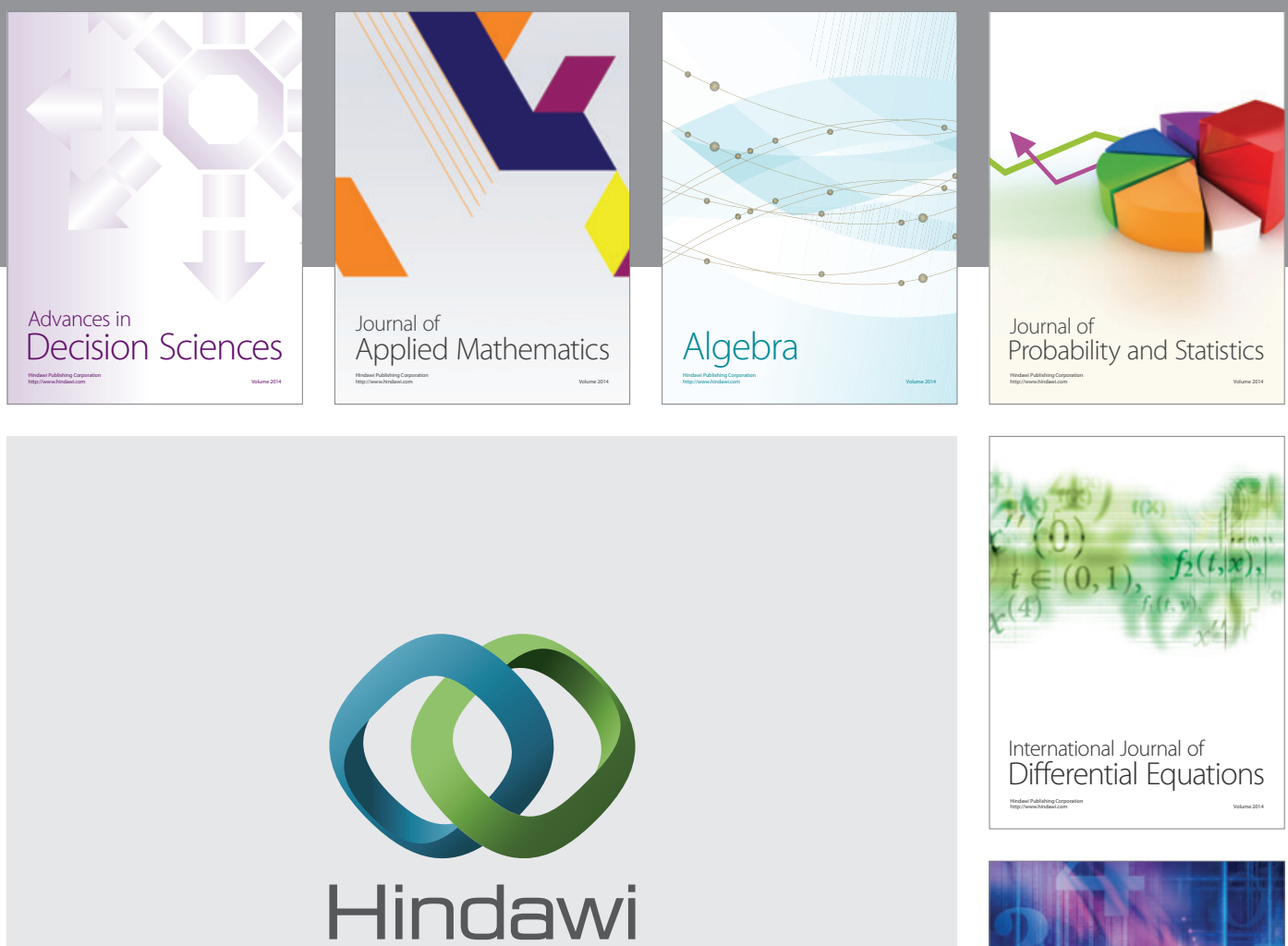

Submit your manuscripts at http://www.hindawi.com
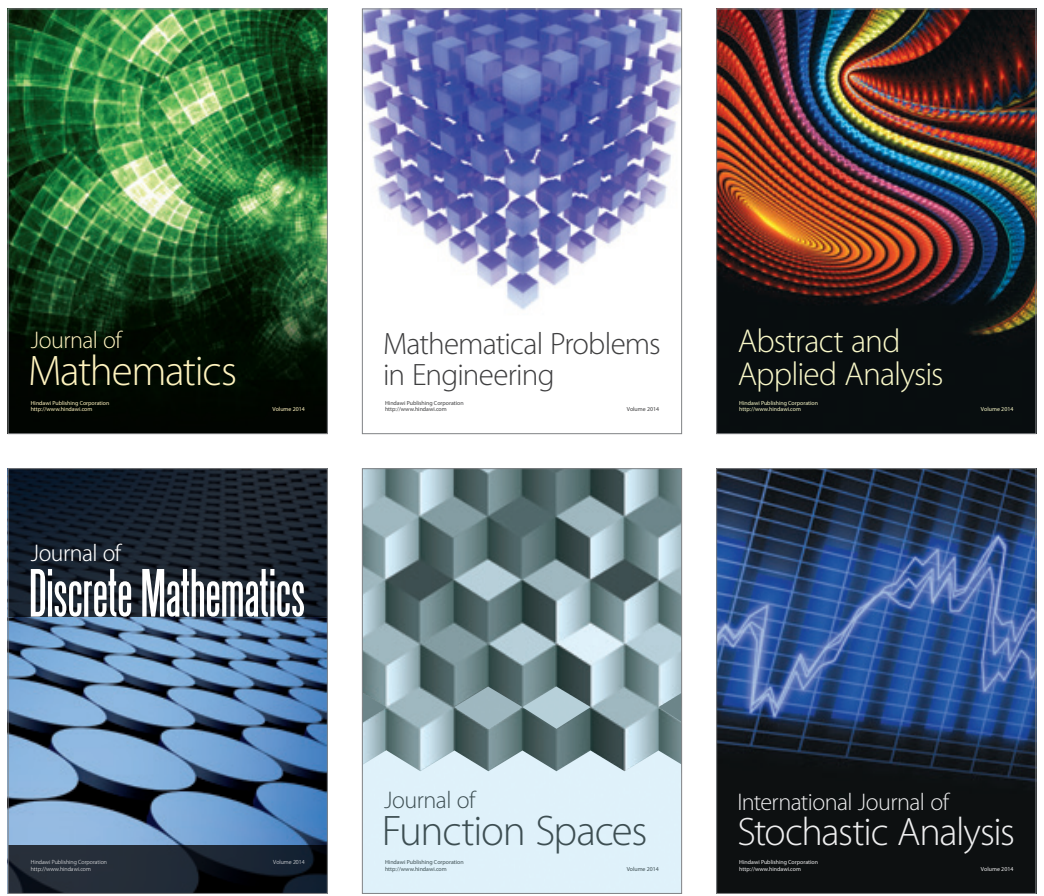

Journal of

Function Spaces

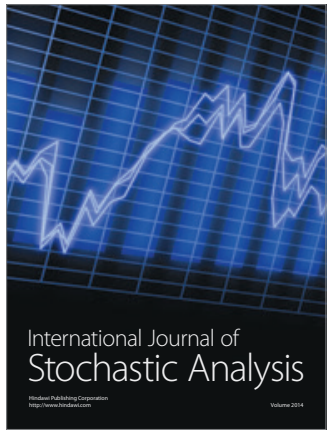

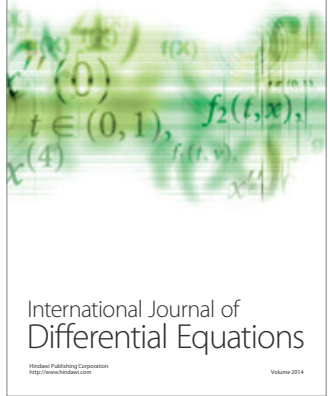
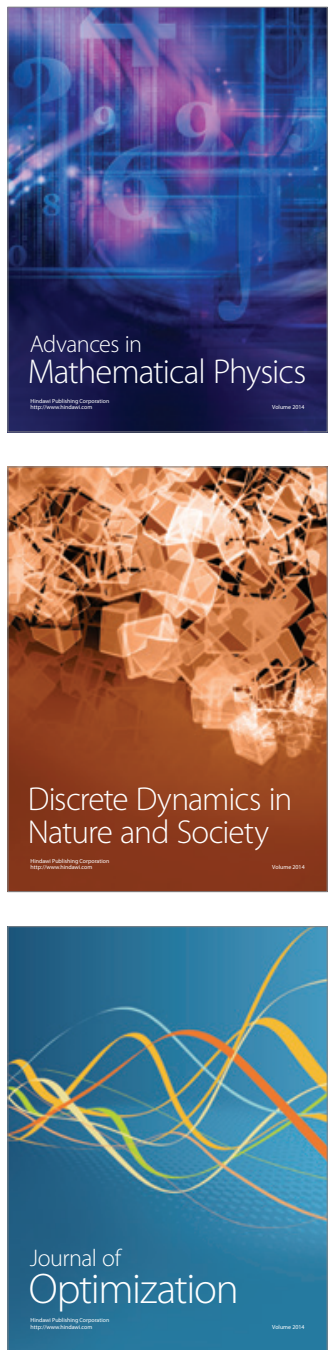